\title{
Spotting imprints of dark matter in the extragalactic Fermi sky with photon counts statistics
}

\author{
Hannes-S. Zechlin* ${ }^{*}$, Silvia Manconi ${ }^{a b}$ and Fiorenza Donato ${ }^{a b}$ \\ ${ }^{a}$ Istituto Nazionale di Fisica Nucleare, Sezione di Torino, via P. Giuria, 1, I-10125 Torino, Italy \\ ${ }^{b}$ Dipartimento di Fisica, Università di Torino, via P. Giuria, 1, I-10125 Torino, Italy \\ E-mail: zechlin@to.infn.it
}

The dissection of the extragalactic gamma-ray background (EGB) into point sources and diffuse components is a valuable tool to search for new physics such as dark matter. In the recent past, it has been shown that statistical analysis methods can excel the sensitivity of classic source detection algorithms with regard to population studies. In this contribution, we analyze the eightyear Fermi-LAT data between 1 and $10 \mathrm{GeV}$ by considering 1-point photon counts statistics. We aim at resolving the population of extragalactic point sources and decomposing the diffuse component into Galactic foreground emission and isotropic diffuse background emission. For the first time, the analysis is employed to incorporate a potential contribution from annihilating dark matter (DM), investigating the sensitivity reach of 1-point photon counts statistics for the DM thermally-averaged self-annihilation cross section $\langle\sigma v\rangle$. We find that the sensitivity of 1-point statistics is competitive with upper limits recently obtained by other indirect detection methods.

7th Fermi Symposium 2017

15-20 October 2017

Garmisch-Partenkirchen, Germany

\footnotetext{
*Speaker.
} 